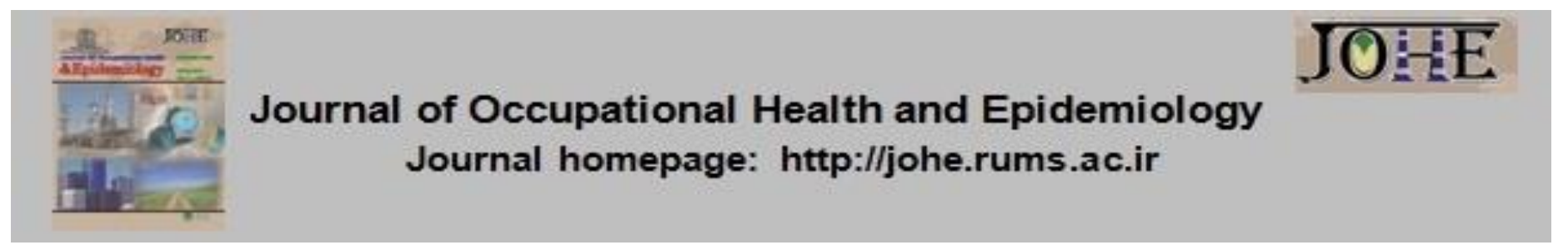

\title{
Anthropometric Dimensions of Lor Students for the Ergonomic Design of Khorramabad School Equipment, Iran (2018)
}

\author{
Rajab Rashidi * \\ - Associate Prof, Dept. of Occupational Health Engineering, Health and Nutrition Research Center, School of Health, Lorestan \\ University of Medical Sciences, Khorramabad, Iran.
}

\section{Article Info}

* Corresponding author:

Rajab Rashidi,

E-mail:

rashidi.r@lums.ac.ir

\section{Article history}

Received: May 2021

Accepted: Jul 2021

10.52547/johe.10.2.67

Print ISSN: $2251-8096$ Online ISSN: 2252-0902

Peer review under responsibility of Journal of Occupational Health and Epidemiology
Citation: Rashidi R. Anthropometric Dimensions of Lor Students for the Ergonomic Design of Khorramabad School Equipment, Iran (2018). JOHE 2021; 10(2):67-74.

\section{Abstract}

Background: Students' physical health, performance, and comfort can be improved by designing equipment according to the needs of their body dimensions. This study aimed to establish a comprehensive database of anthropometric dimensions of students in Khorramabad, Lorestan, Iran.

Materials and Methods: This descriptive study was conducted to estimate 22 anthropometric students' dimensions in a sample of 4872 randomly selected students who belonged to the Lor ethnic group. The anthropometric dimensions were measured by a digital caliper and a standardized anthropometric instrument. Data were analyzed using a t-test, and the results were reported in tabular form.

Results: The results showed that elementary school students' body dimensions were larger in girls, except for chest depth, abdominal depth, and buttock-knee length. Anthropometric estimates of middle school students indicated that most anthropometric dimensions of girls were larger than boys; for example, the average height in girls with $1531.87 \pm 72.84$ was greater than boys with $1528.16 \pm 101.79 \mathrm{~mm}$. Findings in high school students showed that most anthropometric parameters of boys were larger than girls. Also, the t-test showed a significant difference between the means of all measured parameters except the variable of buttock-knee length in male and female students ( $P$ $<0.05)$.

Conclusions: Given the anthropometric differences obtained in this study, it can be concluded that in designing school equipment, especially desks and chairs, it is necessary to pay special attention to age, gender, level of education, and ethnicity of students in particular.

Keywords: Anthropometry, Ergonomics, Student.

\section{Introduction}

Students' physical health, performance, and comfort can be improved by designing equipment according to the needs of the human body dimensions. Nowadays, backache has gained public attention as an important health problem among school children [1, 2, 3, 4]. In the US, about $\$ 25$ million is spent on treating students' nonspecific backaches caused by the nonappropriation of bodily dimensions to the chairs and desks used in classrooms. The use of poorly designed furniture, especially desks and tables, that fails to account for the users' anthropometric characteristics has a negative influence on human health. Students' sitting posture is influenced not only by the activities performed in the classroom but also by their anthropometric measures and school furniture designs. Therefore, one of the most important concerns of ergonomists is to design and produce instruments that match people's bodily dimensions in order to decrease 
the adverse effects, thus increasing work output and efficiency $[5,6,7,8]$.

According to official statistics, the student population in Iran amounts to 20 million, while it is estimated to be one billion globally. However, it seems that no serious and persistent attention has been paid to classrooms, whether nationally or globally, thus neglecting students' health. Various studies have shown that individuals' postures during childhood and adolescence deeply affect the way they sit and stand for a lifetime. Indeed, the longer these early postures are maintained, they are more difficult to be corrected later on [9, $10,11]$. Students spend a great deal of their time in classrooms in sitting positions. Static postures and sitting in a crookbacked manner for long periods can put great physical strains on muscles, tendons, and vertebrae in particular.

Anthropometric measures vary among nations and ethnic groups and change over time depending on variations in populations and environmental conditions [3, 6]. Hence, it has been recommended to use students' anthropometric data to design school supplies and equipment. In order to design the equipment for students (e.g., chairs and desks) according to technical and health principles, it is necessary to aid the designers by providing accurate data about the anthropometric dimensions of the students residing in different geographical regions [6, 7]. One of the most comprehensive anthropometric studies was conducted on students aging 11-13 years in the US in 1975, in which 87 bodily features and dimensions were measured, except for the popliteal height and the buttock-popliteal fossa length [12, 13]. In other countries in Europe and America, the necessary relevant standards have been prepared. Among the international standards approved by the World Health Organization (WHO), those provided by the US National Center for Health Statistics (NCHS) can be pointed out. This center has specified the standards of bodily dimensions for different age groups, which can be used in designing equipment $[14,15,16]$. In Iran, a survey was designed in1977 to compare the height and weight of 3270 students aging 6-14 years in urban and rural areas of Shiraz. Another anthropometric study was carried out in Mazandaran province to measure 17 bodily parameters of 1758 students aged 6-11 years; further, the means and percentiles of the anthropometric dimensions of male and female students were compared. This research objective was to provide the necessary data for designing students' chairs and desks [17, 18]. In another anthropometric survey on children less than 10 years old in Qazvin city, the height and weight of boys and girls were less than the global averages reported by WHO [19]. In another study performed on students in Kashan, emphasis was put on reliance on the necessary standards obtained from domestic anthropometric data rather than foreign standards [20].

At present, few countries have domestic anthropometric databases; thus, other countries have to use their data in case of need, which is scientifically unacceptable. The current study aims to develop a comprehensive database of the anthropometric dimensions of students from the Lor ethnic group residing in Khorramabad. Comparison of the anthropometric measures of different student populations could help design chairs, desks, and other school supplies.

\section{Materials and Methods}

This is a descriptive study conducted on Lor students in Khorramabad in primary, middle, and high schools in the public or non-profit sectors in 2018.

Given the standard variance and the coefficient of variation in similar populations, the appropriate sample size for each class, education level, and sex was determined. In the following formula, the values of $z, \delta$, and $d$ were equal to $1.96,150$, and 6 , respectively. As a result, the sample size was estimated at 2401 people based on the variable of students' height with a standard deviation of 150 according to the formula below [21].

$N=\frac{z^{2} \times \sigma^{2}}{d^{2}}=2401$

Given the design effect, the estimated sample size was multiplied by two $(n=4802)$. Yet, the total sample size was increased to 4872 students with equal numbers of male and female students. Inclusion criteria included all Lor students of different educational levels living in Khorramabad, and exclusion criteria included non-Lor students and those with physical development disorders. Cluster sampling was used in the study; accordingly, each of the three training areas of Khorramabad city was considered as a cluster, and all schools in each cluster were included in the study. Samples were selected randomly from male and female students by a proportional allocation.

The following anthropometric parameters were recorded: age (years), weight $(\mathrm{kg})$, stature, standing eye height, shoulder height, standing elbow height, chest depth, abdominal depth, arm length, forearm length, maximum forearm-forearm breadth, elbow-elbow breadth, shoulder breadth, hip breadth, thigh thickness, the thickness of both thighs measured together, sitting height, sitting eye 
height, sitting elbow height, popliteal height, knee height, buttock-popliteal length, and buttock-knee length. The anthropometric dimensions were measured (in millimeters) by male and female assistants using a digital caliper and a standardized anthropometric instrument designed by Lahmi et al. [21]. Additionally, the students were weighed by Samsung digital scales with an accuracy of \pm 5 grams. The accuracy and reliability of the utilized devices were assessed prior to the measurements. It should be noted that all students were wearing light clothes and were barefoot during the measurements. Besides, the data recorded for each student was the mean of three trials.

Ethics approval was obtained from the officials of Khorramabad University of Medical Sciences and Education. After entering the data into the computer, a t-test was used to compare the variables by gender at a significant level ( $P$ $<0.005)$. Given that percentiles express the real concept of dimensional scattering within the design range, in order to use the data in the design of different percentile equipment, anthropometric dimensions of students were determined. Finally, the results were expressed in a table as averages and standard deviations.

\section{Results}

This cross-sectional study was performed on 4872 male and female students to determine the anthropometric measurements of Lor students in Khorramabad. The average weight and height of male students were $43.05 \mathrm{~kg}$ with a maximum of $114 \mathrm{~kg}$ and minimum $15 \mathrm{~kg}$ and $1499.43 \mathrm{~mm}$ height with a maximum 1900 and minimum 1020 mm. The average weight and height of female students were $41.75 \mathrm{~kg}$ with a maximum of 97 and a minimum of $14 \mathrm{~kg}$ and $1458 \mathrm{~mm}$ height with a maximum of 1760 and a minimum of $1020 \mathrm{~mm}$, respectively. Standard deviation and statistical percentages were calculated as $5 \%$ to $99 \%$.

Table 1 provides the comparison of the mean of anthropometric variables in male and female students. As can be seen, there is a significant difference between the mean of all measured parameters except the variable of buttock-popliteal length in male and female students.

The results also show that the average anthropometric dimensions of the chest depth and abdominal depth in female students are larger than boys; these dimensions in girls were 176.89 and $172.19 \mathrm{~mm}$ compared to boys with 170.93 and $158.99 \mathrm{~mm}$, respectively.

Table 1. Mean \pm SD, min, and max of the anthropometric dimensions in the two genders (in millimeters)

\begin{tabular}{|c|c|c|c|c|c|c|c|c|c|}
\hline \multirow{2}{*}{$\begin{array}{c}\text { Statistical index } \\
\text { variables }\end{array}$} & \multicolumn{4}{|c|}{ Male students } & \multicolumn{4}{|c|}{ Female students } & \multirow{2}{*}{$\begin{array}{l}\text { T-test } \\
\text { results }\end{array}$} \\
\hline & Mean & SD & Min & Max & Mean & SD & Min & Max & \\
\hline Weight (kg) & 43.05 & 16.69 & 15 & 114 & 41.75 & 14.81 & 14 & 97 & 0.004 \\
\hline Stature & 1499.43 & 189.86 & 1020 & 1900 & 1458 & 154.29 & 1020 & 1760 & 0.001 \\
\hline Standing eye height & 1384.72 & 192.62 & 880 & 1790 & 1337.19 & 156.22 & 760 & 1650 & 0.001 \\
\hline Shoulder height & 1233.13 & 175.85 & 640 & 1600 & 1197.08 & 142.58 & 640 & 1480 & 0.001 \\
\hline Standing elbow height & 933.70 & 132.21 & 590 & 1220 & 883.93 & 106.32 & 600 & 1180 & 0.001 \\
\hline Chest depth & 170.93 & 28.19 & 90 & 290 & 176.89 & 31.94 & 80 & 318 & 0.001 \\
\hline Abdominal depth & 158.99 & 30.17 & 80 & 370 & 172.19 & 33.49 & 60 & 398 & 0.001 \\
\hline Arm length & 304.92 & 45.69 & 130 & 490 & 298.33 & 40.80 & 116 & 458 & 0.001 \\
\hline Forearm length & 395.01 & 58.67 & 230 & 530 & 378.65 & 50.35 & 148 & 557 & 0.001 \\
\hline $\begin{array}{l}\text { Maximum forearm-forearm } \\
\text { breadth }\end{array}$ & 359.07 & 50.49 & 200 & 570 & 315.06 & 41 & 116 & 540 & 0.001 \\
\hline Elbow-elbow breadth & 347.15 & 50.78 & 220 & 540 & 343.90 & 46.32 & 188 & 550 & 0.001 \\
\hline Shoulder breadth & 340.02 & 51.98 & 190 & 540 & 330.16 & 44.34 & 110 & 580 & 0.001 \\
\hline Hip breadth & 284.09 & 47.57 & 100 & 450 & 276.11 & 44.09 & 140 & 467 & 0.001 \\
\hline Thigh thickness & 107.13 & 26.56 & 37 & 310 & 75.01 & 21.75 & 22 & 203 & 0.001 \\
\hline $\begin{array}{l}\text { The thickness of both } \\
\text { thighs measured together }\end{array}$ & 194.57 & 40.21 & 97 & 360 & 185.05 & 40.14 & 94 & 396 & 0.001 \\
\hline Sitting height & 786.95 & 95.79 & 480 & 1000 & 753.93 & 82.66 & 440 & 920 & 0.001 \\
\hline Sitting eye height & 674.99 & 95.60 & 390 & 890 & 633.37 & 78.70 & 340 & 860 & 0.001 \\
\hline Sitting elbow height & 209.50 & 35.32 & 80 & 460 & 177.51 & 30.90 & 90 & 320 & 0.001 \\
\hline Popliteal height & 384.33 & 53.04 & 230 & 530 & 353.78 & 31.77 & 210 & 580 & 0.001 \\
\hline Knee height & 462.05 & 64.54 & 240 & 620 & 428.12 & 47.03 & 245 & 598 & 0.001 \\
\hline Buttock-popliteal length & 380.90 & 59.61 & 190 & 570 & 382.89 & 58.55 & 220 & 576 & 0.2 \\
\hline Buttock-knee length & 478.74 & 72.60 & 280 & 650 & 487.67 & 68.22 & 252 & 670 & 0.001 \\
\hline
\end{tabular}

Table 2 presents the different percentiles of anthropometric dimensions of male and female students. Percentiles are very important statistical indicators in developing tools and equipment and are widely used in design engineering. As can be seen, in the 95th percentile, as one of the most important percentiles in equipment design, all dimensions, except chest depth and abdominal depth, in male students have larger values than females. 
Table 2. Percentile values of different anthropometric dimensions of students in the two genders (in millimeters)

\begin{tabular}{|c|c|c|c|c|c|c|c|c|c|c|c|c|}
\hline \multirow{2}{*}{$\begin{array}{c}\text { Percentiles } \\
\text { variables }\end{array}$} & \multicolumn{6}{|c|}{ Male students } & \multicolumn{6}{|c|}{ Female students } \\
\hline & $1^{\text {st }}$ & $5^{\text {th }}$ & $10^{\text {th }}$ & $50^{\text {th }}$ & $95^{\text {th }}$ & $99^{\text {th }}$ & $1^{\text {st }}$ & $5^{\text {th }}$ & $10^{\text {th }}$ & $50^{\text {th }}$ & $95^{\text {th }}$ & $99^{\text {th }}$ \\
\hline Weight (kg) & 17 & 20 & 22 & 41 & 72 & 85 & 17.68 & 20 & 23 & 42 & 66 & 77.32 \\
\hline Height & 1130 & 1190 & 1230 & 1490 & 1770 & 1836 & 1110 & 1180 & 1220 & 1500 & 1660 & 1700 \\
\hline $\begin{array}{c}\text { Standing eye } \\
\text { height }\end{array}$ & 1000 & 1070 & 1120 & 1370 & 1660 & 1720 & 980 & 1050 & 1100 & 1380 & 1540 & 1580 \\
\hline $\begin{array}{c}\text { Standing } \\
\text { shoulder height }\end{array}$ & 890 & 940 & 990 & 1230 & 1480 & 1540 & 880 & 940 & 980 & 1240 & 1380 & 1420 \\
\hline $\begin{array}{c}\text { Standing elbow } \\
\text { height }\end{array}$ & 660 & 710 & 750 & 940 & 1120 & 1170 & 640 & 690 & 720 & 910 & 1020 & 1070 \\
\hline Chest depth & 120 & 130 & 140 & 170 & 220 & 240 & 114.68 & 130 & 139 & 175 & 230 & 257 \\
\hline $\begin{array}{c}\text { Abdominal } \\
\text { depth }\end{array}$ & 110 & 120 & 130 & 150 & 220 & 250 & 105.68 & 124 & 133 & 170 & 230 & 270.32 \\
\hline Arm length & 210 & 230 & 240 & 300 & 370 & 390 & 200 & 230 & 243 & 305 & 354 & 380 \\
\hline Forearm length & 280 & 300 & 310 & 400 & 480 & 500 & 260 & 293 & 310 & 390 & 447 & 32.466 \\
\hline $\begin{array}{c}\text { Maximum } \\
\text { forearm-forearm } \\
\text { breadth }\end{array}$ & 244.4 & 280 & 300 & 360 & 440 & 480 & 228.67 & 250.40 & 264 & 314 & 380 & 420 \\
\hline $\begin{array}{c}\text { Elbow-elbow } \\
\text { breadth }\end{array}$ & 250 & 270 & 280 & 340 & 430 & 490 & 250 & 272 & 386 & 340 & 424 & 468 \\
\hline $\begin{array}{c}\text { Shoulder } \\
\text { breadth }\end{array}$ & 240 & 260 & 270 & 340 & 420 & 450 & 236 & 256 & 270 & 337 & 396 & 420 \\
\hline Hip breadth & 190 & 210 & 220 & 280 & 360 & 390 & 187.68 & 205 & 217 & 279 & 350 & 380 \\
\hline Thigh thickness & 55 & 70 & 80 & 100 & 150 & 180 & 35 & 43 & 48 & 74 & 110 & 136.33 \\
\hline $\begin{array}{c}\text { The thickness } \\
\text { of both thighs } \\
\text { measured } \\
\text { together }\end{array}$ & 120 & 140 & 150 & 190 & 270 & 310 & 114 & 130 & 140 & 180 & 260 & 305.96 \\
\hline Sitting height & 570 & 630 & 660 & 790 & 940 & 970 & 550 & 610 & 630 & 770 & 860 & 890 \\
\hline $\begin{array}{c}\text { Sitting eye } \\
\text { height }\end{array}$ & 460 & 510 & 550 & 670 & 820 & 850 & 450 & 490 & 520 & 650 & 740 & 770 \\
\hline $\begin{array}{c}\text { Sitting elbow } \\
\text { height }\end{array}$ & 120 & 150 & 170 & 210 & 270 & 290 & 100 & 130 & 140 & 180 & 230 & 250 \\
\hline Popliteal height & 270 & 300 & 310 & 390 & 460 & 480 & 280 & 310 & 320 & 350 & 410 & 440 \\
\hline Knee height & 323.2 & 350 & 370 & 470 & 550 & 570 & 316 & 345 & 360 & 438 & 60.496 & 520 \\
\hline $\begin{array}{c}\text { Buttock- } \\
\text { popliteal length }\end{array}$ & 250 & 280 & 300 & 380 & 470 & 500 & 259.68 & 283 & 300 & 390 & 469 & 500 \\
\hline $\begin{array}{c}\text { Buttock-knee } \\
\text { length }\end{array}$ & 320 & 350 & 380 & 480 & 580 & 600 & 335.68 & 364 & 386 & 507 & 580 & 602.32 \\
\hline
\end{tabular}

Table 3 shows the anthropometric dimensions of male and female students at different levels of education. As can be seen, the average growth of anthropometric dimensions in girls often occurs in middle school, while it occurs in high school in males. Further, the average weight and height in middle school girls are $45.93 \mathrm{~kg}$ and $1531.87 \mathrm{~mm}$ respectively, while in boys, the values are $44.06 \mathrm{~kg}$ and $1528.16 \mathrm{~mm}$, respectively, which are lower than the anthropometric measurements of girls

Table 3. Anthropometric estimation of body dimensions among male and female students in different education levels

\begin{tabular}{|c|c|c|c|c|c|c|c|c|c|c|c|c|c|}
\hline \multirow{2}{*}{\multicolumn{2}{|c|}{$\begin{array}{c}\text { Statistical index } \\
\text { variable }\end{array}$}} & \multicolumn{4}{|c|}{ Primary school } & \multicolumn{4}{|c|}{ Junior high school } & \multicolumn{4}{|c|}{ High school } \\
\hline & & Mean & SD & Min & Max & Mean & SD & Min & Max & Mean & SD & Min & Max \\
\hline \multirow{2}{*}{$\begin{array}{l}\text { Weight } \\
\text { (kg) }\end{array}$} & Male & 29.76 & 9.53 & 15 & 71 & 44.06 & 12.54 & 22 & 92 & 58.88 & 11.59 & 30 & 114 \\
\hline & Female & 29.08 & 8.94 & 14 & 73 & 45.93 & 11.13 & 23 & 94 & 54.52 & 9.49 & 36 & 97 \\
\hline \multirow{2}{*}{ Height } & Male & 1321.73 & 107.99 & 1020 & 1660 & 1528.16 & 101.79 & 1270 & 1810 & 1699.31 & 75.42 & 1380 & 1900 \\
\hline & Female & 1308.09 & 109.33 & 1020 & 1660 & 1531.87 & 72.84 & 1310 & 1720 & 1590.26 & 54.78 & 1310 & 1760 \\
\hline \multirow{2}{*}{$\begin{array}{l}\text { Standing } \\
\text { eye height }\end{array}$} & Male & 1204.35 & 108.81 & 880 & 1540 & 1413.85 & 102.05 & 1150 & 1700 & 1587.66 & 78.41 & 1150 & 1790 \\
\hline & Female & 1186.20 & 113.48 & 760 & 1550 & 1411.92 & 71.38 & 1200 & 1610 & 1470.17 & 55.85 & 1130 & 1650 \\
\hline \multirow{2}{*}{$\begin{array}{c}\text { Standing } \\
\text { shoulder } \\
\text { height }\end{array}$} & Male & 1069.51 & 104.87 & 640 & 1400 & 1261.64 & 93.91 & 1030 & 1520 & 1415.82 & 70.22 & 1020 & 1600 \\
\hline & Female & 1060.67 & 105.69 & 640 & 1390 & 1264.40 & 65.95 & 1030 & 1420 & 1318.9 & 51.33 & 1150 & 1480 \\
\hline \multirow{2}{*}{$\begin{array}{l}\text { Standing } \\
\text { elbow } \\
\text { height }\end{array}$} & Male & 811.42 & 83.12 & 590 & 1100 & 956.18 & 70.36 & 790 & 1160 & 1069.26 & 51.22 & 890 & 1220 \\
\hline & Female & 785.80 & 82.66 & 600 & 1070 & 929.53 & 52.84 & 720 & 1070 & 972.65 & 43.62 & 820 & 1180 \\
\hline \multirow{2}{*}{$\begin{array}{l}\text { Chest } \\
\text { depth }\end{array}$} & Male & 152.22 & 20.98 & 90 & 230 & 171.84 & 23.53 & 120 & 290 & 193.57 & 21.70 & 140 & 270 \\
\hline & Female & 154.47 & 22.31 & 80 & 250 & 189.50 & 28.60 & 105 & 312 & 195.45 & 27.11 & 108 & 318 \\
\hline \multirow{2}{*}{$\begin{array}{l}\text { Abdominal } \\
\text { depth }\end{array}$} & Male & 147.98 & 26.36 & 80 & 260 & 159.44 & 31.24 & 110 & 300 & 172.46 & 28.45 & 110 & 370 \\
\hline & Female & 154.71 & 27.83 & 60 & 297 & 186.04 & 34.03 & 102 & 355 & 183.54 & 29.51 & 100 & 398 \\
\hline
\end{tabular}




\begin{tabular}{|c|c|c|c|c|c|c|c|c|c|c|c|c|c|}
\hline \multirow{2}{*}{$\begin{array}{c}\text { Arm } \\
\text { length }\end{array}$} & Male & 266.53 & 32.11 & 140 & 380 & 309.38 & 28.34 & 130 & 400 & 349.42 & 23.10 & 280 & 490 \\
\hline & Female & 265.50 & 32.16 & 130 & 380 & 317.05 & 26.72 & 119 & 458 & 325.31 & 28.96 & 116 & 430 \\
\hline \multirow{2}{*}{$\begin{array}{c}\text { Forearm } \\
\text { length }\end{array}$} & Male & 342.72 & 36.47 & 230 & 450 & 401.10 & 34.46 & 270 & 510 & 455.63 & 25.51 & 320 & 530 \\
\hline & Female & 334.01 & 38.19 & 148 & 452 & 406.73 & 31.84 & 151 & 557 & 413.30 & 27.29 & 289 & 486 \\
\hline \multirow{2}{*}{$\begin{array}{l}\text { Maximum } \\
\text { forearm- } \\
\text { forearm } \\
\text { breadth }\end{array}$} & Male & 328.99 & 42.85 & 200 & 470 & 356.89 & 37.47 & 260 & 480 & 398.25 & 40.43 & 290 & 570 \\
\hline & Female & 288.42 & 32.30 & 207 & 420 & 337.54 & 39.74 & 116 & 501 & 331.29 & 31.86 & 158 & 540 \\
\hline \multirow{2}{*}{$\begin{array}{c}\text { Elbow- } \\
\text { elbow } \\
\text { breadth }\end{array}$} & Male & 324.73 & 46 & 220 & 500 & 342.66 & 47.05 & 230 & 540 & 378.53 & 42.65 & 270 & 540 \\
\hline & Female & 315.25 & 39.68 & 188 & 462 & 361.98 & 42.97 & 262 & 511 & 366.12 & 36.31 & 260 & 550 \\
\hline \multirow{2}{*}{$\begin{array}{l}\text { Shoulder } \\
\text { breadth }\end{array}$} & Male & 300.61 & 36.06 & 190 & 450 & 336.93 & 33.82 & 240 & 440 & 391.46 & 32.05 & 240 & 540 \\
\hline & Female & 295.10 & 35.37 & 110 & 420 & 348.46 & 35.11 & 139 & 580 & 360.31 & 26.79 & 247 & 478 \\
\hline \multirow{2}{*}{$\begin{array}{c}\text { Hip } \\
\text { breadth }\end{array}$} & Male & 249.32 & 36.77 & 100 & 400 & 287.34 & 35.91 & 200 & 410 & 325.02 & 30.73 & 120 & 450 \\
\hline & Female & 242.21 & 33.52 & 140 & 390 & 294.40 & 35.71 & 199 & 467 & 304.80 & 31.10 & 174 & 430 \\
\hline \multirow{2}{*}{$\begin{array}{c}\text { Thigh } \\
\text { thickness } \\
\end{array}$} & Male & 91.97 & 20.87 & 37 & 170 & 107.20 & 23.19 & 50 & 190 & 125.99 & 22.99 & 80 & 310 \\
\hline & Female & 65.42 & 20.74 & 22 & 170 & 85.60 & 21.66 & 30 & 203 & 78.93 & 17.67 & 36 & 141 \\
\hline \multirow{2}{*}{$\begin{array}{c}\text { The } \\
\text { thickness } \\
\text { of both } \\
\text { thighs } \\
\text { measured } \\
\text { together }\end{array}$} & Male & 170.65 & 30.66 & 97 & 290 & 195.95 & 36.51 & 110 & 350 & 223.42 & 33.77 & 140 & 360 \\
\hline & Female & 167.67 & 35.49 & 94 & 358 & 197.19 & 41.48 & 100 & 396 & 197.61 & 36.32 & 113 & 384 \\
\hline \multirow{2}{*}{$\begin{array}{l}\text { Sitting } \\
\text { height }\end{array}$} & Male & 702.79 & 72.59 & 480 & 910 & 798.43 & 52.13 & 580 & 970 & 883.19 & 48.62 & 720 & 1000 \\
\hline & Female & 680.67 & 64.20 & 440 & 890 & 785.22 & 52.51 & 580 & 900 & 822.31 & 34.70 & 670 & 920 \\
\hline \multirow{2}{*}{$\begin{array}{c}\text { Sitting eye } \\
\text { height }\end{array}$} & Male & 590.60 & 60.37 & 390 & 800 & 683.87 & 52.50 & 450 & 860 & 773.38 & 46.22 & 610 & 890 \\
\hline & Female & 563.85 & 61.52 & 340 & 750 & 666.76 & 45.23 & 510 & 780 & 659.39 & 40.46 & 340 & 860 \\
\hline \multirow{2}{*}{$\begin{array}{l}\text { Sitting } \\
\text { elbow } \\
\text { height }\end{array}$} & Male & 185.05 & 29.92 & 80 & 250 & 211.25 & 19.78 & 140 & 270 & 238.69 & 26.99 & 160 & 460 \\
\hline & Female & 162.14 & 28.09 & 90 & 250 & 180.32 & 24.84 & 120 & 260 & 194.76 & 28.73 & 110 & 320 \\
\hline \multirow{2}{*}{$\begin{array}{c}\text { Popliteal } \\
\text { height }\end{array}$} & Male & 337.11 & 35.95 & 250 & 480 & 396.56 & 31.56 & 230 & 530 & 433.98 & 26.63 & 330 & 530 \\
\hline & Female & 334.68 & 29.82 & 210 & 580 & 363.84 & 30.92 & 320 & 460 & 370.15 & 19.89 & 310 & 470 \\
\hline \multirow{2}{*}{$\begin{array}{c}\text { Knee } \\
\text { height }\end{array}$} & Male & 402.59 & 43.14 & 240 & 530 & 478.11 & 35.28 & 360 & 580 & 524.13 & 27.71 & 410 & 620 \\
\hline & Female & 390.97 & 41.90 & 280 & 540 & 452.97 & 31.83 & 317 & 598 & 455.82 & 27.01 & 245 & 556 \\
\hline \multirow{2}{*}{$\begin{array}{l}\text { Buttock- } \\
\text { popliteal } \\
\text { height }\end{array}$} & Male & 331.73 & 45.74 & 190 & 530 & 389.54 & 34.19 & 280 & 510 & 435.65 & 32.96 & 340 & 570 \\
\hline & Female & 331.22 & 42.49 & 220 & 507 & 411.75 & 37.85 & 285 & 576 & 425.83 & 34.10 & 300 & 559 \\
\hline \multirow{2}{*}{$\begin{array}{l}\text { Buttock- } \\
\text { knee } \\
\text { height }\end{array}$} & Male & 413.36 & 49.18 & 280 & 570 & 491.95 & 39.24 & 350 & 610 & 550.31 & 32.17 & 420 & 650 \\
\hline & Female & 426.16 & 74.52 & 260 & 593 & 524.23 & 40.92 & 252 & 670 & 537.10 & 32.71 & 305 & 645 \\
\hline
\end{tabular}

Table 4 provides the different percentiles of anthropometric dimensions for students in both middle and high school. Due to the difference in the anthropometric dimensions of male and female students (Table 3 ), which often occurs in middle and high school, it is important for design engineers to know the percentiles of these two degrees.

Table 4. Percentile values of anthropometric dimensions among students in different education levels

\begin{tabular}{|c|c|c|c|c|c|c|c|c|c|c|c|c|c|c|c|c|}
\hline \multirow{2}{*}{ Variables } & \multirow{2}{*}{ ercentiles } & \multicolumn{5}{|c|}{ Elementary school } & \multicolumn{5}{|c|}{ Junior high school } & \multicolumn{5}{|c|}{ High school } \\
\hline & & $5^{\text {th }}$ & $10^{\text {th }}$ & $50^{\text {th }}$ & $90^{\text {th }}$ & $99^{\text {th }}$ & $5^{\text {th }}$ & $10^{\text {th }}$ & $50^{\text {th }}$ & $90^{\text {th }}$ & $99^{\text {th }}$ & $5^{\text {th }}$ & $10^{\text {th }}$ & $50^{\text {th }}$ & $90^{\text {th }}$ & $99^{\text {th }}$ \\
\hline \multirow{2}{*}{$\begin{array}{c}\text { Weight } \\
\text { (kg) }\end{array}$} & Male & 18 & 20 & 28 & 43 & 60 & 29 & 30 & 42 & 62 & 83.89 & 42 & 45 & 57.5 & 73 & 93.83 \\
\hline & Female & 19 & 20 & 27 & 41 & 59.73 & 30 & 33 & 44 & 60 & 85.69 & 41 & 43 & 54 & 67 & 81.88 \\
\hline \multirow{2}{*}{ Height } & Male & 1150 & 1180 & 1320 & 1460 & 1570 & 1380 & 1401 & 1520 & 1670 & 1780 & 1560 & 1600 & 1710 & 1780 & 1858.30 \\
\hline & Female & 1130 & 1170 & 1300 & 1460 & 1580 & 1400 & 1430 & 1540 & 1620 & 1690 & 1510 & 1520 & 1590 & 1660 & 1710 \\
\hline \multirow{2}{*}{$\begin{array}{l}\text { Standing } \\
\text { eye height }\end{array}$} & Male & 1030 & 1060 & 1200 & 1340 & 1450 & 1265.5 & 1290 & 1410 & 1559 & 1660 & 1448.50 & 1490 & 1595 & 1680 & 1750 \\
\hline & Female & 1000 & 1040 & 1180 & 1340 & 1450 & 1290 & 1320 & 1420 & 1500 & 1570 & 1390 & 1400 & 1470 & 1540 & 1600 \\
\hline \multirow{2}{*}{$\begin{array}{c}\text { Standing } \\
\text { shoulder } \\
\text { height }\end{array}$} & Male & 900 & 930 & 1070 & 1200 & 1298.4 & 1120 & 1140 & 1250 & 1390 & 1488.90 & 1290 & 1330 & 1420 & 1490 & 1578.30 \\
\hline & Female & 900 & 930 & 1060 & 1200 & 1310 & 1150 & 1180 & 1270 & 1340 & 1400 & 1240 & 1260 & 1320 & 1380 & 1440 \\
\hline \multirow{2}{*}{$\begin{array}{l}\text { Standing } \\
\text { elbow } \\
\text { height }\end{array}$} & Male & 680 & 700 & 810 & 910 & 1010 & 850 & 870 & 950 & 1050 & 1120 & 980 & 1010 & 1070 & 1130 & 1190 \\
\hline & Female & 660 & 680 & 780 & 900 & 980 & 840 & 860 & 930 & 1000 & 1050 & 910 & 920 & 970 & 1030 & 1088.80 \\
\hline \multirow{2}{*}{$\begin{array}{l}\text { Chest } \\
\text { depth }\end{array}$} & Male & 120 & 130 & 150 & 180 & 220 & 140 & 150 & 170 & 200 & 248.90 & 160 & 170 & 190 & 220 & 250 \\
\hline & Female & 120 & 129 & 150 & 185 & 210.73 & 150 & 156 & 187 & 224.9 & 274.69 & 152.60 & 165 & 193 & 232 & 261.76 \\
\hline \multirow{2}{*}{$\begin{array}{l}\text { Abdominal } \\
\text { depth }\end{array}$} & Male & 120 & 120 & 140 & 180 & 248.4 & 120 & 130 & 150 & 200 & 278.90 & 140 & 150 & 170 & 200 & 260 \\
\hline & Female & 116 & 123 & 150 & 190 & 239.73 & 141 & 150 & 190 & 230 & 306.90 & 140 & 150 & 180 & 219 & 267.76 \\
\hline \multirow{2}{*}{ Arm length } & Male & 220 & 230 & 260 & 300 & 358.4 & 270 & 280 & 310 & 350 & 388.90 & 310 & 320 & 350 & 370 & 418.30 \\
\hline & Female & 218.35 & 228 & 263 & 307 & 358.38 & 278 & 284 & 319 & 347 & 384.38 & 285 & 298 & 326 & 355 & 407.76 \\
\hline \multirow{2}{*}{$\begin{array}{c}\text { Forearm } \\
\text { length }\end{array}$} & Male & 290 & 300 & 340 & 390 & 428.4 & 350 & 360 & 400 & 450 & 480 & 420 & 430 & 460 & 480 & 510 \\
\hline & Female & 273.70 & 290 & 334 & 385 & 420 & 352.55 & 370 & 410 & 440 & 472.69 & 367 & 380 & 415 & 446 & 472.88 \\
\hline \multirow{2}{*}{$\begin{array}{l}\text { Maximum } \\
\text { forearm- } \\
\text { forearm } \\
\text { breadth }\end{array}$} & Male & 260 & 270 & 330 & 380 & 440 & 300 & 310 & 350 & 410 & 450 & 340 & 350 & 400 & 450 & 500 \\
\hline & Female & 240 & 250 & 286 & 330 & 373.2 & 283 & 292 & 332 & 390 & 456.90 & 280 & 292 & 330 & 370 & 406.76 \\
\hline
\end{tabular}




\begin{tabular}{|c|c|c|c|c|c|c|c|c|c|c|c|c|c|c|c|c|}
\hline \multirow{2}{*}{$\begin{array}{c}\text { Elbow- } \\
\text { elbow } \\
\text { breadth }\end{array}$} & Male & 260 & 270 & 320 & 380 & 468.4 & 280 & 290 & 340 & 410 & 490 & 320 & 330 & 370 & 430 & 500 \\
\hline & Female & 260 & 270 & 310 & 370 & 430 & 299.55 & 313 & 356.50 & 420.90 & 489.69 & 316 & 325 & 364 & 410 & 469.52 \\
\hline \multirow{2}{*}{$\begin{array}{l}\text { Shoulder } \\
\text { breadth }\end{array}$} & Male & 250 & 260 & 300 & 350 & 400 & 280 & 290 & 330 & 380 & 420 & 340 & 350 & 390 & 430 & 480 \\
\hline & Female & 245 & 254 & 290 & 342.3 & 399.2 & 298 & 309 & 345.50 & 390 & 437.14 & 315 & 330 & 360 & 390 & 418 \\
\hline \multirow{2}{*}{$\begin{array}{c}\text { Hip } \\
\text { breadth }\end{array}$} & Male & 200 & 210 & 240 & 300 & 350 & 240 & 250 & 280 & 330 & 388.90 & 280 & 290 & 325 & 360 & 408.30 \\
\hline & Female & 195 & 201.4 & 240 & 284.3 & 340.92 & 244 & 250 & 290 & 340 & 393.45 & 250 & 269 & 303 & 349 & 389.28 \\
\hline \multirow{2}{*}{$\begin{array}{c}\text { Thigh } \\
\text { thickness }\end{array}$} & Male & 60 & 70 & 90 & 120 & 150 & 80 & 80 & 100 & 140 & 178.90 & 100 & 100 & 120 & 150 & 200 \\
\hline & Female & 38 & 43 & 60 & 96 & 120 & 55 & 60 & 84 & 110 & 151.38 & 50 & 57 & 78 & 100 & 130 \\
\hline \multirow{2}{*}{$\begin{array}{c}\text { The } \\
\text { thickness } \\
\text { of both } \\
\text { thighs } \\
\text { measured } \\
\text { together }\end{array}$} & Male & 130 & 140 & 164 & 210 & 270 & 140 & 160 & 190 & 240 & 308.90 & 180 & 187 & 220 & 270 & 328.30 \\
\hline & Female & 120 & 130 & 160 & 212 & 283.2 & 144.55 & 152 & 190 & 259.90 & 310.69 & 144.60 & 156 & 192 & 245 & 314.40 \\
\hline \multirow{2}{*}{$\begin{array}{l}\text { Sitting } \\
\text { height }\end{array}$} & Male & 600 & 620 & 700 & 780 & 850 & 720 & 730 & 800 & 860 & 940 & 800 & 820 & 890 & 950 & 980 \\
\hline & Female & 580 & 600 & 680 & 760 & 847 & 690 & 720 & 790 & 850 & 880 & 770 & 780 & 820 & 870 & 900 \\
\hline \multirow{2}{*}{$\begin{array}{l}\text { Sitting eye } \\
\text { height }\end{array}$} & Male & 490 & 510 & 590 & 670 & 738 & 610 & 620 & 680 & 750 & 828.90 & 690 & 717 & 780 & 830 & 870 \\
\hline & Female & 460 & 490 & 560 & 650 & 710 & 580 & 610 & 670 & 720 & 770 & 630 & 650 & 700 & 740 & 800 \\
\hline \multirow{2}{*}{$\begin{array}{l}\text { Sitting } \\
\text { elbow } \\
\text { height } \\
\end{array}$} & Male & 130 & 150 & 190 & 220 & 248 & 180 & 190 & 210 & 240 & 260 & 200 & 200 & 240 & 270 & 300 \\
\hline & Female & 110 & 120 & 160 & 200 & 237 & 140 & 150 & 180 & 220 & 240 & 150 & 160 & 190 & 230 & 260 \\
\hline \multirow{2}{*}{$\begin{array}{c}\text { Popliteal } \\
\text { height }\end{array}$} & Male & 280 & 300 & 340 & 380 & 440 & 350 & 360 & 400 & 440 & 480 & 390 & 400 & 430 & 470 & 500 \\
\hline & Female & 300 & 310 & 330 & 370 & 430 & 340 & 340 & 350 & 410 & 450 & 350 & 350 & 360 & 400 & 438.80 \\
\hline \multirow{2}{*}{$\begin{array}{c}\text { Knee } \\
\text { height }\end{array}$} & Male & 340 & 350 & 400 & 460 & 518 & 420 & 430 & 480 & 520 & 560 & 480 & 490 & 520 & 560 & 590 \\
\hline & Female & 330 & 340 & 385 & 450 & 507 & 404 & 415 & 450 & 490 & 543 & 415 & 425 & 456 & 490 & 519.52 \\
\hline \multirow{2}{*}{$\begin{array}{l}\text { Buttock- } \\
\text { popliteal } \\
\text { length }\end{array}$} & Male & 260 & 270 & 330 & 390 & 450 & 330 & 350 & 390 & 440 & 470 & 380 & 390 & 435 & 480 & 518.30 \\
\hline & Female & 270 & 280 & 330 & 387 & 457.7 & 350 & 367 & 410.5 & 457.90 & 506.83 & 370 & 383 & 428 & 468.80 & 504.88 \\
\hline \multirow{2}{*}{$\begin{array}{l}\text { Buttock- } \\
\text { knee } \\
\text { length }\end{array}$} & Male & 340 & 350 & 410 & 480 & 540 & 430 & 440 & 490 & 540 & 580 & 498.50 & 510 & 550 & 590 & 628.30 \\
\hline & Female & 350 & 360 & 420 & 500 & 555.8 & 459 & 479 & 528.8 & 571.80 & 616.83 & 485 & 500 & 537 & 577.80 & 613 \\
\hline
\end{tabular}

\section{Discussion}

In a community-based descriptive study, 4872 male and female students of Lor ethnicity in Khorramabad who met the necessary criteria for inclusion were studied to determine 22 anthropometric dimensions used in designing equipment needed by students in schools. In order to use this anthropometric data in designing school equipment, in addition to the mean and standard deviation of each anthropometric dimension of students, the maximum and minimum dimensions were determined. Also, different statistical percentiles, including $5,50,95$, and 99 percentiles widely applied by design engineers in designing tools and equipment used by students, were calculated for different anthropometric dimensions. Comparison of the measured anthropometric dimensions among elementary school students indicated that the anthropometric dimensions of male students were greater than those of females, except for chest depth, abdominal depth, and buttock-knee length. For instance, the means of standing height and sitting height were 1321.73 and $702.79 \mathrm{~mm}$, respectively, among the male students. These measures were respectively obtained as 1308.09 and $680.67 \mathrm{~mm}$ among female students. Additionally, the minimum and maximum height were 1020 and $1660 \mathrm{~mm}$, respectively, for male and female students. The results showed no significant differences between male and female students with regard to the estimated percentiles of anthropometric dimensions. In other words, the obtained sizes were quite close in almost all calculated percentiles. Moreover, the anthropometric dimensions of the elementary school students in Lorestan province were compared to the data obtained in similar studies in Ilam and Mazandaran provinces. The results showed that the bodily dimensions of male and female students residing in Khorramabad were greater than those of the students residing in Ilam and Mazandaran. Therefore, it can be concluded that anthropometric dimensions in different Iranian ethnicities are different. Nonetheless, the anthropometric estimations obtained in this study are almost equal to those published by WHO $[18,19,20]$.

The findings of this study regarding the anthropometric estimations of junior high school students in Khorramabad indicated the mean values for bodily dimensions, such as weight, height, standing shoulder height, chest depth, abdominal depth, arm length, forearm length, elbow-elbow breadth, shoulder breadth, hip breadth, the thickness of both thighs measured together, buttock-popliteal length, and buttockknee length, to be greater among female students compared to male students. This might be attributed to the earlier pubescence among female students and must be taken into account in designing school desks and chairs [9, 10]. According to various percentiles of the anthropometric dimensions of male and female students at the junior high school level, variables 
such as weight, height, standing eye height, arm length, forearm length, and standing shoulder height were greater among female students compared to male students up to the 50th percentile, while the same variables were greater among male students at the 90th and 99th percentiles. Moreover, the anthropometric estimations of standing elbow height, maximum forearm-forearm breadth, sitting height, sitting eye height, sitting elbow height, popliteal height, and knee height were greater among male students compared with female ones. A remarkable point concerning the anthropometric percentiles of students at the junior high school level was that hip breadth, buttock-popliteal length, and buttock-knee length, mostly used in designing chair seats, were greater among female students in various percentiles. Further, the mean height was higher among female students compared to males at the junior high school level $(1531.87 \mathrm{~mm})$, unlike the elementary school level $(1528.16 \mathrm{~mm})$. As mentioned above, this can be explained by earlier pubescence among female students. Comparing the measured anthropometric dimensions of male and female students at the junior high school level in Khorramabad with other similar studies revealed noticeable differences. Accordingly, the mean height of Lor females at this age range was greater than that of females from Hong Kong (1508) but lower compared to English females (1555) [18].

The present study also showed all measured anthropometric parameters to be greater in male students, except for chest depth and abdominal depth. Accordingly, the mean height was 1699.31 $\mathrm{mm}( \pm 75.42)$ among male students and 1590.26 $( \pm 54.78)$ among female ones. Further comparison at the high school level showed that, except for chest depth and abdominal depth being a little greater in size among female students, all other anthropometric dimensions were greater among male students. This difference might be due to musculoskeletal growth among male students in this age range. The anthropometric measurements obtained among high school students in the present study were different from those in similar studies performed on the same age range participants. Accordingly, the mean height of male and female high school students in Khorramabad was greater in those from Hong Kong but lower in those from the UK [18].

In one study, anthropometric indices of height, weight, seating height, seating depth, and seating width of 300 girls and boys aged 18-25 years in southern Thailand were examined by Klamklay et al. (2008). The results indicated a significant difference in the weight, structure, and body dimensions of the subjects, which should be considered for the design of school chairs [22].

Another study showed that the proportion of the furniture dimensions in the schools to the anthropometric dimensions of the students was not optimal. Therefore, it is necessary to consider anthropometric differences in designing a suitable chair for students [23].

The available studies are applicable according to their methodologies, i.e., applying their results to the general Iranian population would have some limitations. Therefore, it is necessary to be up-todated with the existing anthropometric tables to produce a comprehensive table for Iranian anthropometric dimensions [24].

One of the limitations of this study is its large volume of samples, thus coordinating with schools in the study community and transporting the measuring instruments and equipment.

Since the design starts and ends with humans, an appropriate comprehensive database of human anthropometric dimensions is recommended to be created for the relevant designing activities. These results may help design school furniture for Lor students. This study can be conducted in other Iranian ethnic and age groups to achieve a complete bank of anthropometric dimensions required by designers of various equipment.

\section{Conclusion}

This study shows that the anthropometric dimensions of female students in middle school are often larger than male students; also, in high school, boys have larger anthropometric dimensions. It is necessary to pay special attention to students' age and education level in designing school supplies, particularly desks and chairs. Also, the designs should be targeted at specific populations, given the considerable differences between male and female students' body dimensions. Since racial differences are an important factor, designers need to pay attention to anthropometric differences among various ethnic groups.

\section{Acknowledgement}

The authors would like to express their gratitude to the individuals and organizations that cooperated in this study, especially Lorestan University of Medical Sciences.

Conflict of interest: None declared. 


\section{References}

1. Dhara PC, Khaspuri G, Sau SK. Complaints arising from a mismatch between school furniture and anthropometric measurements of rural secondary school children during classwork. Environ Health Prev Med 2009; 14(1):36-45.

2. Tunay M, Melemez K. An analysis of biomechanical and anthropometric parameters on classroom furniture design. Afr J Biotechnol 2008; 7(8):1081-6.

3. Jones GT, Watson KD, Silman AJ, Symmons DP, Macfarlane GJ. Predictors of low back pain in British schoolchildren: a population-based prospective cohort study. Pediatrics 2003; 111(4 pt 1):822-8.

4. Jeffries LJ, Milanese SF, Grimmer-Sommers KA. Epidemiology of adolescent spinal pain: a systematic overview of the research literature. Spine (Phila Pa 1976) 2007; 32(23):2630-7.

5. Hoy D, Bain C, Williams G, March L, Brooks $P$, Blyth $F$, et al. A systematic review of the global prevalence of low back pain. Arthritis Rheum 2012; 64(6):2028-37.

6. Milanese S, Grimmer K. School furniture and the user population: an anthropometric perspective. Ergonomics 2004; 47(4):416-26.

7. Limon S, Valinsky L, Ben-Shalom Y. Children at risk: risk factors for low back pain in the elementary school environment. Spine (Phila $\mathrm{Pa}$ 1976) 2004; 29(6):697-702.

8. Masiero S, Carraro E, Celia A, Sarto D, Ermani $M$. Prevalence of nonspecific low back pain in schoolchildren aged between 13 and 15 years. Acta Paediatr 2008; 97(2):212-6.

9. Preedy VR. Handbook of Anthropometry: Physical Measures of Human form in Health and Disease. 1st ed. New York, United States: Springer-Verlag; 2012.

10. Arefi MF, Pouya AB, Poursadeqiyan M. Investigating the match between anthropometric measures and the classroom furniture dimensions in Iranian students with health approach: A systematic review. J Educ Health Promot 2021; 10:38.

11. Pheasant S, Haslegrave CM. Bodyspace: Anthropometry, Ergonomics, and the Design of Work. 3rd ed. Milton Park, Abingdon, United Kingdom: Taylor \& Francis; 2005.

12. Olsen TL, Anderson RL, Dearwater SR, Kriska AM, Cauley JA, Aaron DJ, et al. The epidemiology of low back pain in an adolescent population. Am J Public Health 1992; 82(4):606-8.
13. Manouchehri H, Moradpour $\mathrm{P}$, Mououdi MA, Aga-Rafiei E. Designing Ergonomic Furniture Based on Students Anthropometry Attributes; College of Agriculture and Natural Resources, University of Tehran. Iran J Ergon 2020; 8(3):70-84

14. de Onis M, Onyango AW, Borghi E, Garza C, Yang $\mathrm{H}$, WHO Multicentre Growth Reference Study Group. Comparison of the World Health Organization (WHO) Child Growth Standards and the National Center for Health Statistics/WHO international growth reference: implications for child health programmes. Public Health Nutr 2006; 9(7):942-7.

15. McDowell MA, Fryar CD, Ogden CL, Flegal KM. Anthropometric reference data for children and adults: United States, 2003-2006. Natl Health Stat Report 2008; (10):1-48.

16. Salvarzi E, Choobineh A, Jahangiri $M$, Keshavarzi S. Facial anthropometric measurements in Iranian male workers using Digimizer version 4.1.1.0 image analysis software: a pilot study. Int J Occup Saf Ergon 2018; 24(4):570-6.

17. Ramin Sabet $M$, Sadeghi F, Safari $M$. Anthropometry and indices of Iranian workers body size to design a workstations. 1st ed. Tehran: Asar Sobhan; 2013.

18. Falahati,M, Zokaei M, Sadeghi Naeini H, Moradi G. Determination of Variables and Static Anthropometric Indicators for Classroom Chair Design. Iran Occup Health J 2013; 10(2):99.

19. Motiei Langrudi SH. A survey of height and weight of children younger than 10 living in Qazvin city. J Inflamm Dis 1997; 1(2):39-46.

20. Ershadi A. Height \& weight of 6 years old pupils Kashan. J Inflamm Dis 1998; 2(2):58-64.

21. Taifa IW, Desai DA. Anthropometric measurements for ergonomic design of students' furniture in India. Eng Sci Technol Int J 2016; 20(1):232-9.

22. Klamklay J, Sungkhapong A, Yodpijit N, Patterson PE. Anthropometry of the southern Thai population. Int J Ind Ergon 2008; 38(1):111-8.

23. Seyedi F, Dalfardi M, Eftekharvaghefi SH. Assessment of Accommodation of Anthropometric Dimensions with Classroom Furniture: Systematic Review Article. J Jiroft Univ Med Sci 2020; 6(2):222-33.

24. Kazemhaghighi M, Saremi M. The situation of anthropometric databank in Iran: a review study. Iran Occup Health J 2017; 14(4):102-95. 